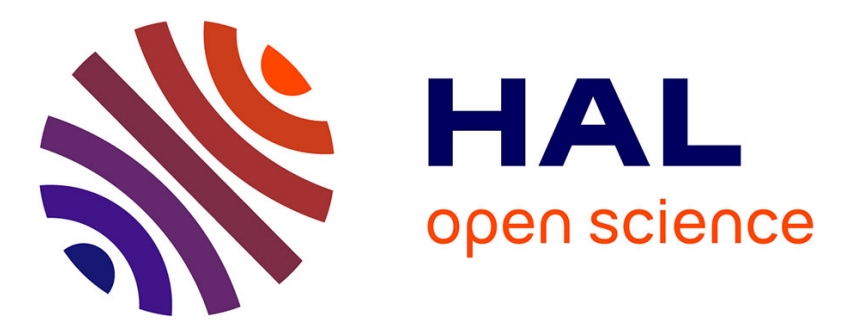

\title{
Évolution de paramètres cinématiques et électromyographiques pendant les différentes phases du lancer de disque: étude préliminaire
}

Daniel Dinu, Jean-Michel Lévêque, Françoise Natta, Henry Vandewalle, P Portero

\section{To cite this version:}

Daniel Dinu, Jean-Michel Lévêque, Françoise Natta, Henry Vandewalle, P Portero. Évolution de paramètres cinématiques et électromyographiques pendant les différentes phases du lancer de disque: étude préliminaire. Science \& Sports, 2004, 19 (4), pp.189-192. 10.1016/j.scispo.2004.02.001 • hal01753799

\section{HAL Id: hal-01753799 \\ https: / hal-insep.archives-ouvertes.fr/hal-01753799}

Submitted on 29 Mar 2018

HAL is a multi-disciplinary open access archive for the deposit and dissemination of scientific research documents, whether they are published or not. The documents may come from teaching and research institutions in France or abroad, or from public or private research centers.
L'archive ouverte pluridisciplinaire HAL, est destinée au dépôt et à la diffusion de documents scientifiques de niveau recherche, publiés ou non, émanant des établissements d'enseignement et de recherche français ou étrangers, des laboratoires publics ou privés. 


\title{
Évolution de paramètres cinématiques et électromyographiques pendant les différentes phases du lancer de disque : étude préliminaire
}

Evolution of kinematical and electromyographic parameters during the different phases of a discus throw: a preliminary study

\section{Dinu ${ }^{\text {a }}$, J.-M. Levêque ${ }^{\text {a }}$, F. Natta ${ }^{\text {b }}$, H. Vandewalle ${ }^{c}$, P. Portero ${ }^{d}$}

* Auteur correspondant. Adresse e-mail : daniel.dinu@insep.fr (D. Dinu).

\begin{abstract}
${ }^{a}$ Laboratoire de biomécanique et physiologie, Institut national du Sport et de l'Education Physique, 11, avenue du Tremblay, 75012 Paris, France

${ }^{\mathrm{b}}$ Laboratoire mouvement action et performance, Institut National du Sport et de l'Education Physique, Paris, France

${ }^{c}$ Département de la formation, Institut national du Sport et de l'Education Physique, Paris, France

d Institut de myologie, groupe hospitalier Pitié-Salpêtrière, 83, boulevard de l'Hôpital, 75651 Paris 13, France
\end{abstract}

(Article publié dans : Science \& Sports, 2004, vol.19, nº4, pp. 189-192)

\section{Résumé}

Introduction. - Analyser l'influence de la masse du disque (1,7 vs $2 \mathrm{~kg}$ ) sur les paramètres cinématiques et l'activité des muscles agonistes au cours du lancer.

Méthodes et résultats. - Quatre lanceurs de haut niveau ont participé à l'étude. Les enregistrements des données cinématiques ont été réalisés au moyen de trois caméras numériques (Panasonic AG-455, 50 Hz) permettant de reconstruire le mouvement en trois dimensions. L'activité électromyographique de surface de 11 muscles a été enregistrée à l' aide d'un appareil numérique embarqué (ME3000P8). La valeur moyenne de quantités électriques pour l'ensemble des muscles analysés semble ne présenter aucune différence significative en fonction de la masse du disque. Cependant, pour certains muscles, l'activité électromyographique intégrée semble plus importante pour le disque léger. Ceci est particulièrement vrai pour les muscles activés durant la deuxième phase de double appui (deltoideus anterior, pectoralis major et biceps brachii). L' analyse cinématique a montré que la durée absolue et relative des différentes phases de lancer et de l'ensemble du jet est la même pour les deux masses du disque (1,7 vs $2 \mathrm{~kg})$.

Conclusion. - Un disque plus léger pourrait être employé pour l'entraînement des athlètes de haut niveau sans altération cinématique du lancer, ce qui pourrait diminuer le risque de blessure lié aux contraintes mécaniques de la charge pendant des périodes d'entraînement et plus particulièrement pour des débutants.

Mots clés : Lancer de disque ; Biomécanique ; Cinématique ; Électromyographie 


\begin{abstract}
Introduction. - Examine how mass of the discus influences the kinematic characteristics and the activity of agonist muscles during throws using three-dimensional videographic and surface electromyography techniques.

Methods and results. — Four throwers of high level participated in the study. Three digitals camcorders (Panasonic AG-455, $50 \mathrm{~Hz}$ ) were used to obtain three-dimensional kinematic data Surface electromyography activity of 11 muscles was recorded using bipolar electrodes. Surface electromyography signals were amplified and recorded by means of a ME3000P8 muscle tester. For some muscles, the integrated surface electromyography mean value, seems to be higher for the $1.7 \mathrm{~kg}$ discus throw and particularly for the ones which were activated during the second double support phase (deltoideus anterior, pectoralis major and biceps brachii muscles). Three-dimensional kinematic analysis provides the same absolute and relative duration for the complete movement and the different phases between the two discus.
\end{abstract}

Conclusion. - A lighter discus might be used for high level training without kinematic alteration of throwing. We assume that this allows decreasing mechanical stress and injury risk during technical learning periods, especially in beginners.

Keywords: Discus throw; Biomechanics; Kinematics; Electromyography

\title{
1. Introduction
}

La performance dans les lancers en général, et en particulier le lancer du disque, dépend principalement des paramètres d'angle et de vitesse d'éjection de l'engin lors du lâcher [5]. L'optimisation de ces paramètres requiert obligatoirement chez le lanceur de disque la maîtrise d' une gestuelle (ou habileté motrice) complexe exécutée dans l'espace restreint qu'est l'aire de lancer du disque. Le lanceur, placé dans les conditions réglementaires, doit enchaîner des actions simultanées de rotation et de translation afin de communiquer au système « lanceur-disque » dans un premier temps puis au disque seul dans un second temps, la plus grande vitesse possible [3]. Il s'avère que les meilleurs lanceurs de disque sont capables de tourner très vite à l'intérieur de l'aire de lancer et d'augmenter la vitesse du disque surtout dans la phase finale de double appui [4].

En condition d'entrainement, de nombreux entraîneurs préconisent l'utilisation d'un disque plus léger que celui utilisé en compétition Senior ( 1,7 vs $2 \mathrm{~kg}$ ). Cette démarche a pour objectif de limiter le risque de blessures lié à la surcharge mécanique induite par la répétition du geste de lancer. Néanmoins aucune donnée expérimentale n'a démontré l'intérêt et l'efficacité de cette situation d'apprentissage. Lancer avec un disque plus léger ne présente un intérêt que dans la mesure où il n'y a pas de modifications importantes des paramètres cinématiques et des patterns de recrutement.

Le principal objectif de cette étude, réalisée avec les quatre meilleurs lanceurs de disque français, est d'analyser l'influence de la masse du disque (1,7 vs $2 \mathrm{~kg})$ sur les paramètres cinématiques et électromyographiques de surface (EMGs) mesurés au cours du lancer de disque.

\section{Méthodologie}

Quatre lanceurs de haut niveau international (meilleure performance : $57 \pm 3 \mathrm{~m}$; âge : $23 \pm 3$ ans ; poids : $108 \pm 19 \mathrm{~kg}$; taille : $190 \pm 6 \mathrm{~cm}$ ) ont participé à l'étude. Les sujets ont exécuté 12 jets dans la même séance de mesure ( 6 avec un disque de $1,7 \mathrm{~kg}$ et 6 avec un disque de $2 \mathrm{~kg}$ ), dans un ordre aléatoire. Les lancers ont été effectués dans une salle de sport avec un filet permettant la réception du disque. La distance de jet (dj) a été calculée en fonction de la vitesse d'éjection $(\mathrm{Ve})$, de la hauteur $(h)$ et de l'angle de lâcher $(\alpha)$ du disque à l'aide des équations du mouvement uniformément accéléré. Seuls les jets égaux à au moins $80 \%$ de la meilleure performance individuelle et avec un angle de déviation de la trajectoire $\beta= \pm 20^{\circ}$, ont été pris en compte dans cette analyse. Les axes du mouvement sont placés dans un référentiel orthonormé. Les axes Z-Y constituent le plan sagittal qui divise le plateau de lancer en deux parties droite et gauche. La norme de la vitesse horizontale $(V x y)$ a été calculée dans le plan horizontal $X-Y$ [2]. L'angle d'éjection $(\alpha)$ est déterminé par la 
norme de la vitesse d'éjection $(V e)$, la norme de la vitesse horizontale $(V x y)$ et de la hauteur $(h)$ du lâcher. L'angle est déterminé par la composante $(\mathrm{Vy})$ de la norme de la vitesse d'éjection $(\mathrm{Ve})$ et la norme de la vitesse horizontale $(V x y)$ dans le plan X-Y (équations 1 et 2) :

$$
\alpha=\arccos \frac{|\mathrm{Vxy}|}{|\mathrm{Ve}|}
$$

$V e=$ norme de la vitesse d'éjection ;

$V x ; V y ; V z=$ composantes de la vitesse d'éjection ;

$V x y=$ norme de la vitesse résultante dans le plan $X-Y$;

$$
\beta=\arccos \frac{|V y|}{|V x y|}
$$

$\alpha=$ angle d'éjection ;

$h=$ hauteur du lâcher ;

$d j=$ distance de jet ;

$\beta=$ angle de déviation de la trajectoire du disque.

Dans cette étude, les forces de frottement, la portance et la traînée ont été négligées. Les enregistrements des données cinématiques ont été réalisés au moyen de trois caméras numériques, (Panasonic AG-455, 50 Hz), permettant de reconstruire le mouvement en trois dimensions par une méthode de DLT (Direct Linear Transformation [3]). Les caméras étaient disposées à trois mètres du plateau et avec un angle de $120^{\circ}$ entre elles par rapport au centre du plateau. Un étalonnage de l'espace de lancer a été réalisé au préalable. Dix-sept marqueurs ont été fixés en regard des principaux centres articulaires de l'athlète [3].

L'approche cinématique, en référence aux travaux de la littérature [1] a permis d'identifier les différentes phases du lancer (Fig. 1) : une phase préparatoire en double appui $\left(\Delta t_{\text {lda }}\right)$; une phase initiale de simple appui ou pivot $\left(\Delta t_{1 s a}\right)$; la phase aérienne ou volte $\left(\Delta \mathrm{t}_{\mathrm{v}}\right)$; une phase finale en simple appui $\left(\Delta \mathrm{t}_{2 s a}\right) 1$ et une phase finale en double appui ( $\left.\Delta t_{2 d a}\right)$ ou phase de lâcher). De plus, cette approche a permis de calculer la vitesse du disque pendant les différentes phases du mouvement.

L'activité EMGs a été enregistrée au moyen d'électrodes bipolaires (Blue Sensor de $15 \mathrm{~mm}$ ) sur neuf muscles; du côté droit (biceps brachii [BB], triceps brachii [TB], deltoideus pars anterior [DA] et pars medialis [DM], pectoralis major [PM], obliquus externus [0E], latissimus dorsi [LD], vastus lateralis [VU, biceps femoris [BF], trapezius pars descendens [TS]) et deux muscles, du côté gauche, (erector lumbar spinae [ES], obliquus internus [0I]).

Les signaux EMGs ont été enregistrés (fréquence d'acquisition de $1000 \mathrm{~Hz}$ ) à l'aide d'un appareil embarqué de type ME3000P8 (méga électronique Ltd, Kuopio, Finlande). Le signal EMGs a été intégré (EMGi) et normalisé par rapport à l'activité EMGs maximale recueillie préalablement au cours d'un test spécifique à chaque muscle étudié.

L'analyse des activités EMGs a porté sur chaque phase et sur la totalité du lancer. Elle prend en compte la durée de chacune des phases (EMGi/dt : l'activité électromyographique intégrée, normalisée et en fonction de temps de l'ensemble des muscles analysés).

Les résultats proposés représentent pour chaque disque (1,7 vs $2 \mathrm{~kg})$ la moyenne des valeurs obtenues pour les qautre athlètes lors de trois de leurs meilleurs jets. 


\section{Résultats}

Nos résultats montrent que les patterns de recrutement électromyographiques des muscles analysés et l'aspect général de l'évolution de la norme de vitesses du disque, sont reproductibles d'un essai à l'autre et en relation avec les phases du lancer (Fig. 1).

L'analyse cinématique a montré que la durée absolue et relative des différentes phases de lancer et de l'ensemble du jet est la même pour les deux masses du disque ( $1,7 \mathrm{vs} 2 \mathrm{~kg}$ ). Les valeurs moyennes de vitesse d'éjection et de distance parcourue par le disque après le lâcher, apparaissent plus grandes pour le disque de $1,7 \mathrm{~kg}$ (Tableau 1). Cette différence semble liée à un gain de vitesse du disque $(1,7 \mathrm{~kg}$ vs $2 \mathrm{~kg})$ plus important pendant la phase finale en double appui $\left(\Delta t_{2 d a}\right)^{\prime}\left(12,93 \pm 1,36 \mathrm{~m} \mathrm{~s}^{-1} \mathrm{vs} 11,04 \pm 1,01 \mathrm{~m} \mathrm{~s}^{-1}\right)$.

La moyenne de quantités électriques (EMGi/dt) pour l'ensemble des muscles analysés semble ne présenter aucune différence significative en fonction de la masse du disque $(1,7 v s 2 \mathrm{~kg})(189 \pm 168 \mathrm{UA}$ vs $192 \pm$ 176 UA) (Tableau 1).

Cependant, pour certains muscles la valeur moyenne de l'EMGi/dt, semble plus importante pour le disque léger et pour d'autres pour le disque lourd. Ceci est vrai, en particulier, pour les muscles qui ont été activés pendant la dernière phase de double appui, BB (158 \pm 100 UA vs $145 \pm 69$ UA), PM (117 \pm 23 UA vs $113 \pm$ $26 \mathrm{UA}), \mathrm{VL}(141 \pm 50 \mathrm{UA}$ vs $140 \pm 45 \mathrm{UA})$.

\section{Discussion}

Ces résultats préliminaires montrent que, quelle que soit la masse du disque, l'allure générale de l'évolution de la vitesse au cours des cinq phases n'est pas modifiée.

Ceci n'est, cependant, pas vrai pour le gain de vitesse dans la phase finale de double appui qui est plus important avec le disque de 1,7 kg. Par ailleurs, la modification de la masse du disque n'a pas d'effet sur la chronologie de la mise en action des muscles (Fig. 1). Enfin, une analyse quantitative permet d'approcher la part plus spécifique de l'activité musculaire dans le mouvement. Par exemple, il a été remarqué que la quantité de EMGi est toujours plus importante pour le DA pendant la phase $\Delta \mathrm{t}_{2 d a}$, alors qu'au contraire, pour le DM, EMGi semble plus important pendant la phase $\Delta \mathrm{t}_{1 \mathrm{sa}}$. La masse du disque n'a donc pas d'effet sur le niveau d'activation musculaire dans cette étude préliminaire.

\section{Conclusion}

Ces résultats suggèrent qu'un disque plus léger pourrait être employé pour l'entraînement des athlètes de haut niveau sans altération cinématique du lancer. Ceci pourrait diminuer le risque de blessure lié aux contraintes mécaniques de la charge pendant des périodes d'entraînement et plus particulièrement pour des débutants. Enfin, il serait nécessaire de compléter l'analyse quantitative en calculant les quantités segmentaires de mouvement pendant le lancer, la translation et la rotation.

\section{Références}

[1] Bartlett RM. The biomechanics of the discus throw. J Sports Sci 1992;10:467-510.

[2] Chow JW, Chae W-S, Crawford MJ. Kinematic analysis of shotputting performed by wheelchair athletes of different medical classes. J Sports Sci 2000;18:321-30.

[3] Dapena J. A method to determine the angular momentum of a human body about three orthogonal axes passing through its center of gravity. JBiomech 1978;11:251-6.

[4] Schlüter W, Nixdorf E. Kinematische Beschreibung und Analyse der Diskuswurftechnik. Leistungssport 1984;6:17-22. 
[5] Stepanek J, Susanka P. Discus throw: Results of a biomechanics study. New Studies in Athletics 1986;1:2536. 
Figure . 1. Exemple d'activité électromyographique de surface (EMGs en $\mathrm{mV}$ ) enregistrée sur dix muscles lors d'un lancer réalisé avec le disque d'une masse de $2 \mathrm{~kg}$. EMGs est représenté au cours des cinq phases caractéristiques du lancer ( $c f$. méthodologie) en fonction de l'évolution du module de la vitesse du disque. Muscles analysés :

- huit muscles droits : biceps brachii (BB), deltoideus pars anterior et pars medialis (DA et DM), pectoralis major, (PM), trapezius pars descendens (TM), obliquus externus (0E), latissimus dorsi (LD), vastus lateralis (VL), biceps femoris (BF) ;

- deux muscles gauches : erector lombaire spinae (ES) et obliquus internus (01).
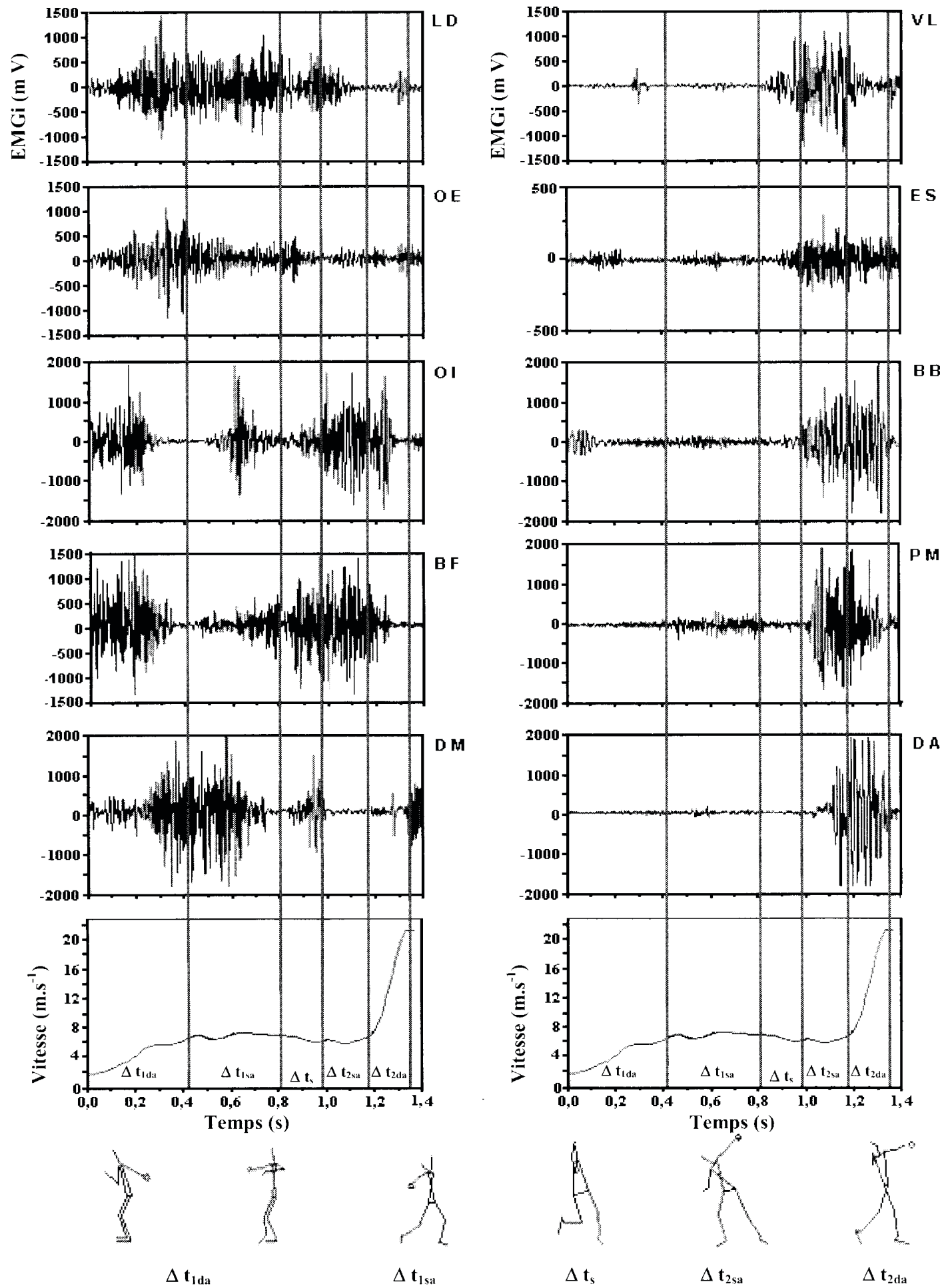

$\Delta \mathbf{t}_{\mathrm{s}}$

$\Delta t_{2 \mathrm{sa}}$

$\Delta \mathbf{t}_{2 \mathrm{da}}$ 


\section{Tableau I}

Paramètres cinématiques et électromyographiques (moyennes et écart type) calculés pour les quatre athlètes avec les deux disques de masses différentes (1,7 vs $2 \mathrm{~kg})$

La durée ( $\Delta \mathrm{t}$, exprimée en valeur absolue (s) et relative $(\%))$ et la variation de vitesse $\left(\Delta \mathrm{V}\right.$, en $\left.\mathrm{m} \mathrm{s}^{-\mathrm{T}}\right)$ sont calculées pour chacune des cinq phases caractéristiques du lancer ( $c f$ méthodologie). $\Sigma E M G i(A U)$ correspond à la sommation des quantités électriques calculées pour tous les sujets sur l'ensemble des muscles analysés au cours du lancer

Paramètres biomécaniques

Valeurs moyennes S1-4

\begin{tabular}{|c|c|c|}
\hline & $1,7 \mathrm{~kg}$ & $2 \mathrm{~kg}$ \\
\hline Vdte $\left(\mathrm{m} \mathrm{s}^{-1}\right)$ & $21,12 \pm 1,06$ & $19,62 \pm 0,46$ \\
\hline$\Delta\left(^{\circ}\right)$ & $36,58 \pm 3,18$ & $39,23 \pm 0,44$ \\
\hline $\operatorname{dj}(\mathrm{m})$ & $47,75 \pm 0,99$ & $42,66 \pm 2,88$ \\
\hline$\Delta \mathrm{t}_{1 \mathrm{da}}(\mathrm{s})$ & $0,43 \pm 0,10$ & $0,45 \pm 0,22$ \\
\hline$\Delta t_{1 d a}(\%)$ & $30,66 \pm 2,47$ & $31,63 \pm 4,74$ \\
\hline$\Delta \mathrm{t}_{1 \mathrm{sa}}(\mathrm{s})$ & $0,46 \pm 0,09$ & $0,46 \pm 0,07$ \\
\hline$\Delta \mathrm{t}_{1 \mathrm{da}}(\%)$ & $32,44 \pm 2,32$ & $31,99 \pm 3,26$ \\
\hline$\Delta \mathrm{t}_{\mathrm{s}}(\mathrm{s})$ & $0,10 \pm 0,05$ & $0,10 \pm 0,04$ \\
\hline$\Delta \mathrm{t}_{\mathrm{s}}(\%)$ & $6,95 \pm 3,96$ & $7,21 \pm 3,08$ \\
\hline$\Delta \mathrm{t}_{2 \mathrm{sa}} \cdot(\mathrm{s})$ & $0,21 \pm 0,04$ & $0,21 \pm 0,03$ \\
\hline$\Delta \mathrm{t}_{2 \mathrm{sa}}(\%)$ & $14,8 \pm 0,77$ & $14,94 \pm 2,15$ \\
\hline$\Delta \mathrm{t}_{2 \mathrm{da}}(\mathrm{s})$ & $0,21 \pm 0,07$ & $0,20 \pm 0,07$ \\
\hline$\Delta \mathrm{t}_{2 \mathrm{da}}(\%)$ & $15,15 \pm 4,21$ & $14,24 \pm 3,12$ \\
\hline $\mathrm{t}_{\mathrm{a}}(\mathrm{s})$ & $1,40 \pm 0,25$ & $1,42 \pm 0,24$ \\
\hline$\Delta \mathrm{Vt}_{1 \mathrm{da}}\left(\mathrm{m} \mathrm{s}^{-1}\right)$ & $5,57 \pm 1,89$ & $5,74 \pm 1,65$ \\
\hline$\Delta \mathrm{Vt}_{1 \mathrm{sa}},\left(\mathrm{m} \mathrm{s}^{-1}\right)$ & $1,60 \pm 1,66$ & $1,83 \pm 1,75$ \\
\hline$\Delta \mathrm{V}_{\mathrm{ts}}\left(\mathrm{m} \mathrm{s}^{-1}\right)$ & $0,27 \pm 1,01$ & $-0,28 \pm 0,98$ \\
\hline$\Delta \mathrm{Vt}_{2 \mathrm{sa}}\left(\mathrm{m} \mathrm{s}^{-1}\right)$ & $0,76 \pm 2,23$ & $1,29 \pm 1,17$ \\
\hline$\Delta \mathrm{Vt}_{2 \mathrm{da}}\left(\mathrm{m} \mathrm{s}^{-1}\right)$ & $12,93 \pm 1,36$ & $11,04 \pm 1,01$ \\
\hline Moyenne EMGi (UA) & $189 \pm 168$ & $192 \pm 176$ \\
\hline
\end{tabular}

